\title{
On the influence of higher order gradient terms on the hydrodynamics of liquid crystals
}

\author{
H. Pleiner and H. Brand \\ Universität Essen, Fachbereich Physik, 4300 Essen, W-Germany \\ (Reçu le 15 juillet 1980, accepté le $1^{\mathrm{er}}$ septembre 1980)
}

\begin{abstract}
Résumé. - L'influence de termes du troisième ordre sur l'hydrodynamique non linéaire est discutée pour différents types de cristaux liquides : les nématiques, les cholestériques, les smectiques $C$ et les discotiques nématiques biaxes. Pour déterminer l'importance expérimentale des contributions nouvelles présentées, nous proposons une expérience statique pour les nématiques.
\end{abstract}

\begin{abstract}
The influence of terms which are of third order is discussed for the nonlinear hydrodynamics of various types of liquid crystals like nematics, cholesterics, smectics $\mathrm{C}$ and biaxial nematic discotics. To test the experimental importance of the presented new contributions we propose a static experiment to be carried out on nematics.
\end{abstract}

1. Introduction. - When dealing with the hydrodynamics of liquid crystals [1-6] one usually takes into account the contributions with the two lowest orders in the wavevector. As it has become obvious in a very recent study by $R$. Combescot [7] for superfluid ${ }^{3} \mathrm{He}-\mathrm{A}$ and $-\mathrm{B}$ it is also necessary to keep third order terms which include gradients of the conserved quantities in the free energy in order to give a microscopically correct description of the so called gauge wheel effect of ${ }^{3} \mathrm{He}-\mathrm{A}[8]$.

It is the purpose of the present letter to investigate the influence of these higher order terms on the hydrodynamics of liquid crystals. We look not only for static effects but we also discuss contributions to the reversible and irreversible currents. By third order terms we mean the following:

In general we have conserved quantities like the density $\rho$, the entropy density $\sigma$ or the density of linear momentum $\mathbf{g}$ and hydrodynamic variables characterizing broken symmetries like $\nabla_{i} n_{j}$, the gradients of the transverse components of the director in nematics or $\nabla_{i} R_{j}$ the gradient of the displacement vector in crystals. All these quantities will be considered to be first order and in most cases one expands the free energy quadratically in the hydrodynamics variables. However, as is discussed in [5] it is necessary to expand to third order in the gradients of the variables characterizing broken symmetries to get systematically derived hydrodynamic equations (which can be applied to all experimentally accessible situa- tions), if the thermodynamic conjugates are inserted into the currents. The expansion to third order is completed by keeping in the free energy gradients of conserved quantities like $\nabla_{i} \rho$ or $\nabla_{i} \sigma$ (which are in our notation of second order). This is motivated by the appropriate procedure in superfluid ${ }^{3} \mathrm{He}$ and leads - as will be shown below - to new measurable effects. The experimental verification of these predicted effects, would on the other hand demonstrate the necessity of including such terms. One should keep in mind, however, that a free energy which is expanded to third order in the above stated sense can never be positive.

Therefore it is important to note that the free energy which is of third order must be completed at least in principle by terms which are of fourth order to ensure positivity of the free energy as a thermodynamic potential.

Furthermore it is important to notice that it is far from clear at present to what order in the wave vector a hydrodynamic theory can be set up because microscopic theories may prove in the future that there exist nonanalytic contributions like $k \ln k$ to the modes thus making obscure already the second order in $k$ in the chosen example.

As in all hydrodynamic theories the wave vectors are restricted by $k l \ll 1$, where $l$ is the greatest microscopic length of the system under consideration.

With all this in mind we turn to the discussion of the influence of third order terms on the hydrodyna- 
mics of nematics, cholesterics, smectics $\mathrm{C}$ and biaxial nematic discotics and to the proposal of an experiment which may evaluate the magnitude of one of the additional coefficients and test the validity of including higher order gradient terms.

2. Nematics. - For nematic liquid crystals we find, the following third order contributions to the free energy

$$
\begin{aligned}
F^{g}=F^{0} & +\lambda^{1 \rho}\left(\partial_{i} n_{i}\right)\left(n_{j} \partial_{j} \rho\right)+\lambda^{2 \rho}\left(\partial_{i} n_{j}\right)\left(n_{i} \partial_{j} \rho\right)+ \\
& +\lambda^{1 \sigma}\left(\partial_{i} n_{i}\right)\left(n_{j} \partial_{j} \sigma\right)+\lambda^{2 \sigma}\left(\partial_{i} n_{j}\right)\left(n_{j} \partial_{j} \sigma\right)
\end{aligned}
$$

where $F^{0}$ contains all the contributions already discussed in $[1,2,5]$ and $n_{j}$ denotes the director of nematic liquid crystals. Thus we obtain four novel static contributions which persist even after a linearization and which couple the director to the gradients of the density and the entropy density respectively.

One further contribution is found in the currents for the director and for the entropy density

$$
\begin{gathered}
Z_{i}=Z_{i}^{0}-\eta_{1} \nabla_{k} n_{k} \delta_{i j}^{\mathrm{tr}} \nabla_{j} T-\eta_{2} \nabla_{k} n_{j} \delta_{i k}^{\mathrm{tr}} \nabla_{j} T \\
j_{i}^{\sigma}=j_{i}^{\sigma^{\mathrm{o}}}+\eta_{1} n_{k} \delta_{i q}^{\mathrm{tr}} \nabla_{k} \nabla_{j} \varphi_{q j}+\eta_{2} \delta_{j k}^{\mathrm{tr}} n_{i} \nabla_{k} \nabla_{p} \varphi_{j p}
\end{gathered}
$$

where $Z_{i}^{0}, j_{i}^{\sigma^{0}}$ denote the currents of the director and of the entropy density which have already been given in $[2,5]$. As it is seen immediately $\eta_{1}, \eta_{2}$ are irreversible terms and it is easily checked that they contribute to hydrodynamic equations to the same order in $k$ as the static contributions given in equation (1).

3. Cholesterics. - As is well known [2-5], cholesteric liquid crystals, which are characterized by an inhomogeneous order parameter in thermodynamic equilibrium [1], have one variable associated with a broken symmetry in addition to the usual conserved quantities. For this type of liquid crystals we find two additional static contribution of third order

$$
\begin{aligned}
F^{g}=F^{0}+\chi_{1} q_{0} p_{k} p_{i} p_{j}\left(\nabla_{i} R_{k}\right)\left(\nabla_{j} \rho\right)+ \\
+\chi_{2} q_{0} p_{k}\left(\nabla_{i} R_{k}\right)\left(\nabla_{j} \sigma\right) p_{i} p_{j}
\end{aligned}
$$

where $\boldsymbol{R}_{\boldsymbol{k}}$ is the displacement of the layers, $q_{0}$ is a pseudoscalar which is associated with the pitch and p denotes the pitch axis. It seems worth to notice that Cholesterics represent the only type of liquid crystals which possess a pseudoscalar $q_{0}$ which allows contributions of the form (4). Such types of contributions are not possible for Smectics A which have only a broken translational symmetry and neither a broken rotational symmetry nor the pseudoscalar which exists in Cholesterics.

In addition to these static contributions we have some dynamic contributions as well

$$
\begin{aligned}
j_{i}^{\sigma} & =q_{0} \lambda_{i m k} \nabla_{m} V_{k}^{n}+j_{i}^{\sigma^{0}} \\
\sigma_{k m} & =-q_{0} \lambda_{i m k} \nabla_{i} T+\sigma_{k m}^{0}
\end{aligned}
$$

where $j_{i}^{\sigma^{0}}$ and $\sigma_{k m}^{0}$ are the currents of entropy density and density of linear momentum as presented in [5] and where

$$
\begin{aligned}
\lambda_{i m k}=\lambda_{1} p_{i} p_{m} p_{k}+\lambda_{2}\left(p_{i} \delta_{m k}^{\mathrm{tr}}+p_{m}\right. & \left.\delta_{i k}^{\mathrm{tr}}\right)+ \\
& +\lambda_{3} \delta_{i m}^{\mathrm{tr}} p_{k} .
\end{aligned}
$$

Contrary to the case of nematics the dynamic contributions to the dynamics of cholesterics are reversible ones.

4. Smectics C. - Smectics $\mathrm{C}$ liquid crystals possess simultaneously a broken rotational symmetry and a broken translational symmetry and form thus a biaxial system.

For the static contributions we find

$$
F^{g}=F^{0}+E_{i j k}\left(\nabla_{i} n_{j}\right)\left(\nabla_{k} \rho\right)+F_{i j k}\left(\nabla_{i} n_{j}\right)\left(\nabla_{k} \sigma\right)
$$

where

$$
\begin{aligned}
& E_{i j k}=E_{1} \delta_{j k}^{3} n_{i}+E_{2} p_{i} \delta_{j k}^{3}+E_{3} n_{k} \delta_{i j}^{3}+E_{4} p_{k} \delta_{i j}^{3} \\
& F_{i j k}=F_{1} \delta_{j k}^{3} n_{i}+F_{2} p_{i} \delta_{j k}^{3}+F_{3} n_{k} \delta_{i j}^{3}+F_{4} p_{k} \delta_{i j}^{3}
\end{aligned}
$$

and where

$$
\delta_{j k}^{3}=\delta_{j k}-n_{j} n_{k}-p_{j} p_{k}
$$

$\left(\left(\nabla_{i} n_{j}\right)\right.$ characterizes the broken rotational symmetry and $n_{j}$ and $p_{k}$ are the preferred directions in smectics C). Thus we find eight additional static terms compared to reference [5]. (Of course, it should be kept in mind that the terms considered in the present letter are third order contributions !).

For the dynamic currents we have

$$
\begin{aligned}
& Y_{i}=Y_{i}^{0}+\zeta_{i k j} \nabla_{k} \nabla_{j} T \\
& j_{i}^{\sigma}=j_{i}^{\sigma^{0}}+\zeta_{i k j} \nabla_{k} \nabla_{j} \varphi_{q j} \delta_{i q}^{3}
\end{aligned}
$$

where

$$
\zeta_{i k j}=\zeta_{1} \delta_{i j}^{3} n_{k}+\zeta_{2} \delta_{i j}^{3} p_{k}
$$

These two contributions emerge in the dissipative regime in the same order in $k$ like the static contributions of equations (8).

5. Biaxial nematic like discotics. - In the last two years it became obvious that discotic liquid crystals, mesophases which consist of disclike molecules, possess as the first man made type of liquid crystals very exciting properties. Very recently the present authors have given a hydrodynamic description of biaxial discotics. As has been shown in [6] it would be very important to synthesize discotic liquid crystals with different broken rotational symmetries, i.e. the orientation of the molecules shows long range order in two different directions $\mathbf{n}$ and $\mathbf{m}$. 
In addition to the results presented in [6] we have the following third order terms in the free energy

$$
\begin{aligned}
F^{g}=F^{0}+ & B_{i j k}^{(\alpha)}\left(\partial_{i} n_{j}\right)\left(\partial_{k} \rho\right)+C_{i j k}^{(\alpha)}\left(\partial_{i} m_{j}\right)\left(\partial_{k} \rho\right)+ \\
& +B_{i j k}^{(\beta)}\left(\partial_{i} n_{j}\right)\left(\partial_{k} \sigma\right)+C_{i j k}^{(\beta)}\left(\partial_{i} m_{j}\right)\left(\partial_{k} \sigma\right)
\end{aligned}
$$

where

$$
\begin{aligned}
B_{i j k}^{(\alpha, \beta)}=n_{i}\left(B_{1}^{(\alpha, \beta)} \delta_{j k}^{3}+\right. & \left.B_{2}^{(\alpha, \beta)} m_{k} m_{j}\right)+ \\
& +n_{k}\left(B_{3}^{(\alpha, \beta)} \delta_{i j}^{3}+B_{4}^{(\alpha, \beta)} m_{i} m_{j}\right)
\end{aligned}
$$

and

$$
C_{i j k}^{(\alpha, \beta)}=m_{i}\left(C_{1}^{(\alpha, \beta)} \delta_{j k}^{3}\right)+C_{2}^{(\alpha, \beta)} m_{k} \delta_{i j}^{3} .
$$

As has been discussed in [6] we have three hydrodynamic variables characterizing broken rotational symmetries in biaxial nematic discotics namely $\delta n_{1}=(\mathbf{n} \times \mathbf{m}) . \delta \mathbf{n}, \delta n_{2}=\delta \mathbf{n} . \mathbf{m}$ and $\delta m=\delta \mathbf{m} .(\mathbf{n} \times \mathbf{m})$.

From equation (11) we find that 12 additional elastic constants are present.

Furthermore there are some irreversible contributions in the currents as well

$$
\begin{aligned}
X_{i}^{(n)} & =X_{i}^{0(n)}+\xi_{i j k}^{(1)} \nabla_{k} \nabla_{j} T \\
X_{i}^{(m)} & =X_{i}^{0(m)}+\xi_{i j k}^{(2)} \nabla_{k} \nabla_{j} T \\
j_{i}^{(\sigma)} & =j_{i}^{(\sigma)}+\nabla_{k} \xi_{j i k}^{(1)} H_{j}^{(n)}+\nabla_{k} \xi_{j i k}^{(2)} H_{j}^{(m)}
\end{aligned}
$$

where

$$
\begin{aligned}
& \xi_{i j k}^{(1)}=\xi_{1}\left(\delta_{i k}^{3} n_{j}+\delta_{i j}^{3} n_{k}\right)+\xi_{2} m_{i}\left(m_{k} n_{j}+m_{j} n_{k}\right) \\
& \xi_{i j k}^{(2)}=\xi_{3}\left(\delta_{i k}^{3} m_{j}+\delta_{i j}^{3} m_{k}\right) .
\end{aligned}
$$

For the definition of $H_{i}^{(n, m)} \mathrm{cf}$. [6]. Thus the dynamics brings along three novel parameters.

6. Possible experimental test. - In this section we would like to propose a static experiment which may yield some information on the order of magnitude and thus of the importance of the additional terms discussed in the present letter so far. For convenience and because this type of liquid crystal has been tested experimentally very thoroughly we consider nematic liquid crystals. Of course corresponding experiments can be carried out equally well on the various other types described here. We look at a planar texture of nematics which is bent upwards as sketched

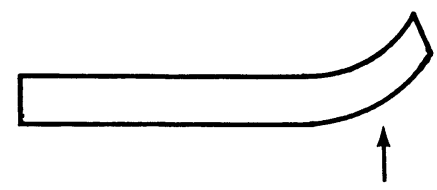

We find for this configuration the equations of state for the chemical potential, the temperature and the thermodynamic conjugates of $\nabla_{i} n_{j}$

$$
\begin{array}{r}
\delta \mu=\zeta_{3} \delta T+\zeta_{4} \delta \rho-\lambda^{1 \rho} \nabla_{j}\left(n_{j} \nabla_{i} n_{i}\right)- \\
-\lambda^{2 \rho} \nabla_{j}\left(n_{i} \nabla_{i} n_{j}\right) \\
\delta T=C_{v}^{-1} T_{0} \delta \sigma+\zeta_{3} \delta \rho-\lambda^{1 \sigma} \nabla_{j}\left(n_{j} \nabla_{i} n_{i}\right)- \\
-\lambda^{2 \sigma} \nabla_{j}\left(n_{i} \nabla_{i} n_{j}\right) \\
\varphi_{i j}=k_{i j k l} \nabla_{l} n_{k}+\lambda^{1 \rho} n_{i} \nabla_{i} \rho+\lambda^{1 \sigma} n_{i} \nabla_{i} \sigma+ \\
+\lambda^{2 \rho} \delta_{i j} n_{k} \nabla_{k} \rho+\lambda^{2 \sigma} \delta_{i j} n_{k} \nabla_{k} \sigma
\end{array}
$$

Equations (14)-(16) can easily be obtained from equations (3.10) of reference [5] and equation (1).

If the proposed experiment is carried out adiabatically $(\delta \sigma=0)$ and under the requirement of incompressibility $(\delta \rho=0)$ we obtain from equations (14)(16)

$$
\begin{aligned}
& \begin{aligned}
\delta \mu & =-\lambda^{1 \rho} \nabla_{j}\left(n_{j} \nabla_{i} n_{i}\right)-\lambda^{2 \rho} \nabla_{j}\left(n_{i} \nabla_{i} n_{j}\right) \equiv \\
& \equiv \frac{1}{\rho_{0}} \delta p-\frac{\sigma_{0}}{\rho_{0}} \delta T
\end{aligned} \\
& \begin{aligned}
\delta T & =-\lambda^{1 \sigma} \nabla_{j}\left(n_{j} \nabla_{i} n_{i}\right)-\lambda^{2 \sigma} \nabla_{j}\left(n_{i} \nabla_{i} n_{j}\right) \\
\varphi_{i j} & =k_{i j k l} \nabla_{l} n_{k} .
\end{aligned}
\end{aligned}
$$

Thus by bending the planar texture of nematics we find across the sample a unique temperature difference $\delta T$ (eq. (18)) and a unique pressure difference $\delta p$ (eq. (17) and (18)) which should be measurable if $\lambda^{1 \rho}, \lambda^{2 \rho}, \lambda^{1 \sigma}$ and $\lambda^{2 \sigma}$ are sufficiently large.

To summarize, we have found various third order contributions, static as well as dynamic ones to the hydrodynamic equations of various types of liquid crystals and we have presented a proposal for an experimental test for some of these novel parameters.

\section{References}

[1] De Gennes, P. G., The Physics of Liquid Crystals (Clarendon, Oxford) 1974

[2] Martin, P. C., Parodi, O., Pershan, P. S., Phys. Rev. A6 (1972) 2401.

[3] Lubensky, T. C., Mol. Cryst. Liq. Cryst. 23 (1973) 99.
[4] Lubensky, T. C., Phys. Rev. A 6 (1972) 452.

[5] Brand, H. and Pleiner, H., J. Physique 41 (1980) 553.

[6] Brand, $H$. and Pleiner, H., submitted for publication.

[7] Combescot, R., preprint may 80.

[8] Liu, M. and Cross, M. C., Phys. Rev. Lett. 43 (1979) 296. 